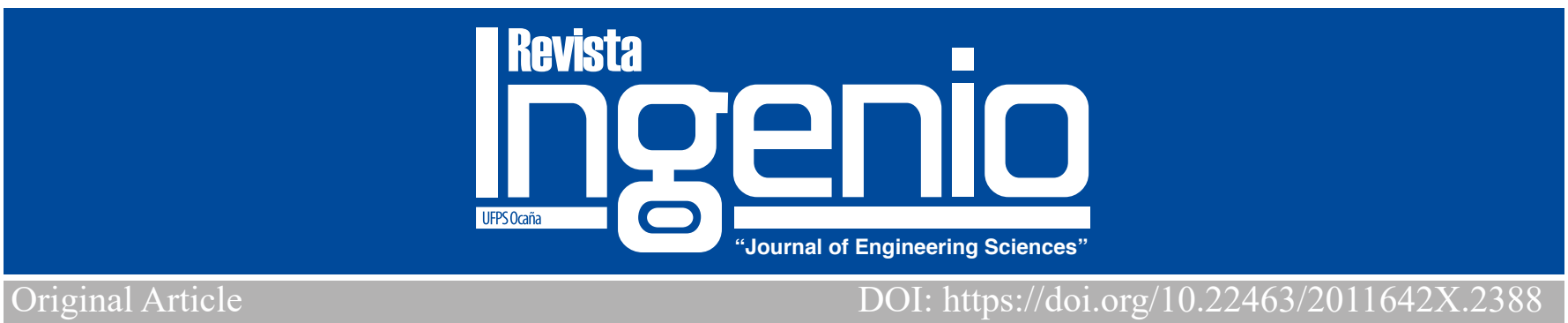

\title{
A topological approach to the study of COVID-19 pandemic: qualitative models for understanding and helping taking decisions
}

Un abordaje topológico en el estudio de la pandemia COVID-19: modelos cualitativos para comprender y ayudar a tomar decisiones

PhD. Jesús Rodríguez-Millán ${ }^{1}$

${ }^{l}$ Grupo de Investigación en Sistemas Dinámicos, División de Estudios de Postgrado de la Facultad de Ingenieria, Universidad de Los Andes, Venezuela, Orcid: https://orcid.org/0000-0003-3771-4608, Email: jrmillan@ula.ve

How to cite: J. Rodriguez-Millán, "A topological approach to the study of COVID-19 pandemic: qualitative models for understanding and helping taking decisions”, Revista Ingenio, 18 (1), pp.41-47, 2021

\section{ABSTRACT}

Keywords:

Epidemics, faults, strategic and tactical models, order of a dynamical system, topologies.
Mathematical models are either strategic, simplified, to study global qualitative properties, or tactic, detailed, appropriate for fine quantitative adjustment to reality. When complex systems interact with their medium or undergo parameter perturbations, they can suffer changes of order making qualitative and quantitative studies difficult. Epidemiological processes allow distinguishing between topological and dynamical alterations, and establishing precedence among them. In this essay we approach COVID-19 this way, to separate topological transformations inducing changes of order in the system, from dynamic transformations themselves. We then develop a visual metaphor, a sequence of images to support a stop-motion, allowing distinguishing the stages, identifying and classifying sceneries, and suggest actions to improve the understanding and control, of the pandemic.

\section{RESUMEN}

Palabras claves:

Epidemias, fallas, modelos estratégicos y tácticos, orden de un sistema dinámico, topologías.
Los modelos matemáticos pertenecen a dos grandes grupos: los estratégicos, simplificados, que estudian las propiedades cualitativas globales y los tácticos, detallados, capaces de ajustes cuantitativos finos a la realidad. Cuando los sistemas complejos interactúan con el medio o sufren variaciones en sus parámetros, pueden sufrir cambios de orden que dificultan sus estudios cualitativos y cuantitativos. Los procesos epidemiológicos permiten separar claramente las alteraciones topológicas de las alteraciones dinámicas y establecer relaciones de precedencia entre ellas. En este ensayo se revisa la pandemia COVID-19 bajo esta óptica, para separar las alteraciones topológicas inductoras de cambios de orden del sistema, de las alteraciones dinámicas propiamente dichas. A partir de ello se desarrolla una metáfora visual, una sucesión de imágenes, que permite distinguir las etapas, identificar y clasificar posibles escenarios, y sugerir acciones a tomar para mejorar la comprensión o el control, de la epidemia.

\section{Introduction}

The construction of models of the reality is a cyclic process that can be roughly sketched as in the commutative diagram of Figure 1. It starts by observing the phenomena to be modeled and collecting relevant data about it. Natural sciences, with all their techniques and methods, are responsible for this first stage. The collected data must then be interpreted and organized in search for patterns and regularities, allowing establishing cause-effect relationships. Mathematics is responsible for both abstract thinking and modeling. Models lead to acting upon reality through technology. Evaluation and reflection complete a first cycle of the process of modeling, and leads to a second cycle of observingthinking-acting-reflecting, which will hopefully sharp our observations, improve the mimetic properties of our models, and refine our technological abilities to modify reality.

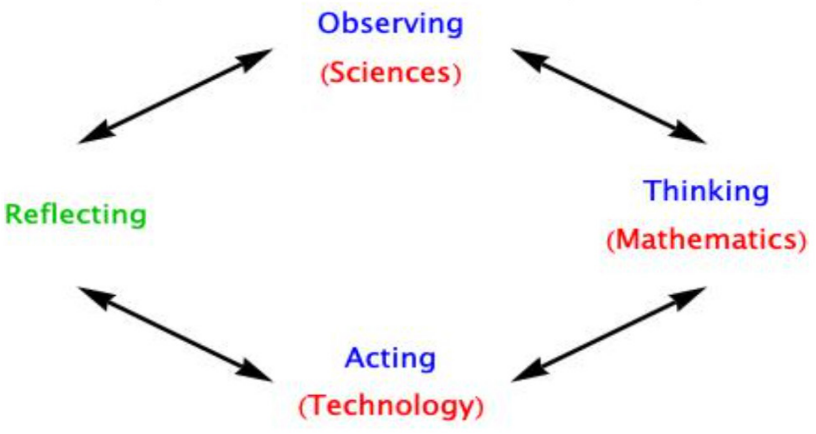

Figure 1. The Observing-Thinking-Acting-Reflecting cycle supporting scientific modeling. 
1.1 Models and modeling

Why do we need models? A good answer to this question could be Huntington's [1] even if it comes from political sciences and not from system's bibliography: "Simplified paradigms or maps are indispensable for human thought and action. On the one hand, we may explicitly formulate theories or models and consciously use them to guide our behavior. Alternatively, we may deny the need for such guides and assume that we will act only in terms of specific "objective" facts, dealing with each case "on its merits". If we assume this, however, we delude ourselves. For in the back of our minds are hidden assumptions, biases, and prejudices that determine how we perceive reality, what facts we look at, and how we judge their importance and merits. We need explicit or implicit models so as to be able to:

1. order and generalize about reality;

2. understand causal relationships among phenomena;

3. anticipate and, if we are lucky, predict future developments;

4. distinguish what is important from what is unimportant; and

5. show us what paths we should take to achieve our goals."

Modeling always involves a trade off between simplicity and accuracy. On the one hand we search for an as simple as possible model trapping the main features of a process to gain a global understanding of it, and on the other hand we need a detailed enough model leading to sharp predictions of the future behavior of the process or to the possibility of controlling it accurately. In general, no single model satisfies both requirements simultaneously, which force us to choose between strategic models and tactical models [2], depending on the main goals of the modeling task. Strategic models are simple enough to trap and display the main qualitative features of the modeled process, whereas tactical models are detailed enough to fit real behavior quantitatively, but at a high computational cost. In most cases, mathematicians privilege strategic models because they use to pursue global qualitative understanding, while engineers or public health providers looking for practical methods and procedures to tackle with everyday problems privilege tactical models.

\subsection{Mathematical Models}

In this paper we will only study systems admitting being modeled as dynamical systems [3-5]. Dynamical systems were invented by I. Newton and G. W. Leibniz during the seventeenth century in connection with celestial mechanics problems [5-6-7]. Newton was so proud of his invention and so conscious of its importance that he dares to affirm "The laws of nature are expressed by differential equations" [4]. During next hundred years mathematicians were occupied integrating particular differential equations, and did not attempt formulating a general theory of differential equations. By the end of the 18th century, it was clear that most differential equations were not solvable in quadrature, and that numerical integration could only provide information about particular solutions in short-term intervals of time. The study of the long-term behavior of solutions had to wait for Poincaré and Lyapunov, who created the qualitative theory of differential equations by the end of the 19th century [4-5].

Dynamical systems may appear either as a differential equation $\dot{\mathrm{x}}_{\mathrm{i}}=\mathrm{f}_{\mathrm{i}}\left(\mathrm{x}_{1}, \ldots, \mathrm{x}_{\mathrm{n}}\right), 1 \leq \mathrm{i} \leq \mathrm{n}$, whose right side do not explicitly depends on the independent variable $t$, and $f_{i}$ is Lipschitz continuous in some domain $U \subseteq \mathbb{R}^{n}$, or as a $\in$ low $\Phi: \mathbb{R} \times X \rightarrow X$ in the space $X$, which assigns to each $\mathrm{x} X$, and to each $\mathrm{t} \in \mathbb{R}$, a point $\Phi(\mathrm{t}, \mathrm{x}) \in X$, satisfying the following three properties: (1) Initial condition: $\Phi(0, x)=x, \forall \quad x \in X$. (2) Simultaneous continuity with respect to both arguments:

$\lim _{\substack{t \rightarrow t_{0} \\ x \rightarrow x_{0}}} \Phi(t, x)=\Phi\left(t_{0}, x_{0}\right), \quad \forall x \in X, \quad$ and

$\forall t \in \mathbb{R}$

(3) Group property: $\Phi\left(\mathrm{t}_{1}, \Phi\left(\mathrm{t}_{2}, \mathrm{x}_{0}\right)\right)=\Phi\left(\mathrm{t}_{1}+\mathrm{t}_{2}, \mathrm{x}_{0}\right)$. The name dynamical systems was introduce by Birkhoff [5], the independent variable $\mathrm{t}$ use to be called time, the solutions $\mathrm{X}_{i}: \mathbb{R} \rightarrow \mathrm{X}$ are called motions, $\mathrm{x}_{1}, \ldots, \mathrm{X}_{\mathrm{n}}$ are the coordinates of a moving point $\mathrm{x}=\left(\mathrm{x}_{1}, \ldots, \mathrm{X}_{\mathrm{n}}\right) \in \mathbb{R}^{n}$, $\mathbb{R}^{n}$ is called the phase space, and the curves $\Phi: \mathbb{R} \rightarrow \mathbb{R}^{n}$, $\Phi(\mathrm{t})=\left(\mathrm{x}_{1}(\mathrm{t}), \ldots, \mathrm{x}_{\mathrm{n}}(\mathrm{t})\right)$, are called the trajectories of the motion [5].

In the Newtonian modeling paradigm the differential equations governing the behavior of a mechanistic system arise from gluing together the mathematical models describing the behavior of the components of the system, with those of the interconnection rules of the components. Contrastingly, the flow approach to dynamical systems had its origin within mathematics, and emerges naturally from global analysis, and the interpretation of trajectories as diffeomorphisms or actions of uniparametric continuous groups on points and subsets of phase spaces. From the modeling point of view, the differential equations and the flow approaches are equivalent, in the sense that any flow is associated to an autonomous differential equation, and any autonomous differential equation generates a 
local flow, which, were it needed, could be turn into a global topologically identical flow by arch-length reparametrization [5-8-9-10].

\subsection{Autonomous dynamical systems}

Dynamical systems modeled by differential equations $\dot{x_{i}}=f_{i}\left(x_{1}, \ldots, x_{n}\right), 1 \leq i \leq \mathrm{n}$, are a mere subset of the set of all differential equations $\dot{x}_{i}=f_{i}\left(\mathrm{t}, x_{1}, \ldots, x_{n}\right), 1 \leq i \leq n$, namely, the subset of differential equations whose vector fields $f_{i}\left(x_{1}, \ldots, x_{n}\right), 1 \leq i \leq \mathrm{n}$, do not explicitly depend on time. So, strictly speaking, the term dynamical system only applies to the family of the autonomous systems, where the independence of the vector fields $f_{i}\left(x_{1}, \ldots, x_{n}\right), 1 \leq i \leq \mathrm{n}$, of time $t$ actually models the time invariance of the laws of nature, without which it would be nearly impossible to model mother nature [4]. Being autonomous has two important consequences: firstly, the trajectories of autonomous systems satisfy the group property of flows [5], which allows to concatenate segments of trajectories; and secondly, if $\Phi(\mathrm{t})$ is a solution of the autonomous system, then so it is $\Phi(\mathrm{t}+\mathrm{s}), \forall \mathrm{s} \in \mathbb{R}[4$, $10]$, which means that trajectories are invariant under translations along time axis. The elementary equation $x=2 \mathrm{tx}$ allows verifying explicitly that the trajectories of nonautonomous differential equations do not satisfy the group property, nor admit time translations. Having said that, and for the sake of the present essay, some additional remarks concerning the concept of dynamical system are still in order.

If we scan some fundamental literature of differential equations and dynamical systems [3-4-5-9-10-11-1213-14-15-16-17] we can observe that both differential equations and flows are always defined on subsets of spaces of constant dimension. Thus, differential equations are defined in: "a certain domain $\mathrm{G}$ of the Euclidean space $\mathbb{R}^{n}$ " in [13], in rectangles, open sets or domains (open connected subsets) $D \subset \mathbb{R}^{n}$ in [10-11-1214-15-16], in an open D of a finite dimension Banach space $\mathrm{E}$ over $\mathrm{K}$ in [17]; whereas flows are define in: "a metric space $R$ " in [13-5], an open set $D \in \mathbb{R}^{n}$ in [10-16-17], or a manifold M [9]. Then, in all cases, the trajectories associated to autonomous differential equations or flows are curves $\Phi: \mathrm{R} \rightarrow D \subset \mathbb{R}^{n}$, such that for all $\mathrm{t} \in \mathbb{R}, \Phi(\mathrm{t})=\left(\phi_{1}(\mathrm{t}), \ldots, \phi_{n}(\mathrm{t})\right) \in \mathbb{R}^{n}$, with $\mathrm{n}$ a fixed natural number. This also holds even in the case of n-dimensional nonautonomous systems if we transform them into autonomous systems of order $n+1$ in the usual way [10].

So, in its present standard form, the theory of dynamical systems cannot cope with "varying order systems", that is to say, systems whose order could (no matter the reason) change, what would be equivalent to posses orbits which under some conditions would be n-dimensional curves, and under another different conditions would be a m-dimensional curves. Yet, such systems exist in many disciplines, and abound in disciplines dealing with complex socio-biological processes or technological processes with sociological implications.

\section{Pandemics, revolutions, bifurcations, catastrophes, and topologies}

By default applied scientists and engineers think in terms of dynamics evolving in $\mathbb{R}^{n}$, and not in terms of topologies, topological structures in more abstract spaces, changes in topological structures and their properties, and the associated concepts of open sets, sets of opens sets, equivalence relations, equivalence classes, coverings, partitions, and so on [18-19-20]. As we have seen, dynamics are modeled through autonomous differential or difference equations, in the case of continuous-time or discrete-time dynamical systems, respectively; yet, these models cannot cope with systems presenting changes of order or dimension, no matter whether they are real processes like mutations [21], epidemics, black outs [20], revolutions [20], or cultural fictions, legends and myths like the Genesis [20], the Dao De Jing, or the Lord of the Rings.

In what follows we will adopt as a leit motiv one of the maxim of physiology, according to which structures precede functions or, equivalently, there does not exist functions without the corresponding anatomical supporting structure. Thus, we could formulate our main thesis the following way: every structural change in the dynamics of a system is preceded by a change in the supporting topological structure of the system. We refer the reader to [20] for a summary of basic supporting concepts of topology that, for space limitations, cannot be included in this essay.

\subsection{Generalities about COVID-19}

Coronavirus disease 2019 (COVID-19), produced by the coronavirus SARS-CoV-2 [22], is an acute infectious respiratory disease, first reported in December 2019 in the city of Wuhan, the capital of Hubei province of China. To date, July 16th 2020, it has transformed into a global ongoing pandemic, affecting 188 countries, 13,589,273 million people worldwide, out of which 7,607,0033 million people have recovered, and 584,989 have died [23]. Evidences suggest that the virus is natural of animal origin, yet the actual origin of the pandemic is unknown [22]. According to the World Health Organization (WHO) neither vaccines nor 
specific antiviral treatments exist yet. WHO declared the COVID-19 outbreak a Public Health Emergency of International Concern on January 30th 2020, and a pandemic on March 11th 2020.

Sensitivity to the coronavirus strongly depends on age. So, of all cases $1 \%$ are under 10 years, and $4 \%$ between 10 and 19 years. In people younger than 50 years the risk of death is less than $0.5 \%$, in those older than 70 it is $8 \%$, and in those older than 80 it is higher than $22 \%$. Most of those who died of COVID-19 had a least one comorbidity like hypertension, diabetes mellitus, and cardiovascular disease. In China men had a death rate of $2.8 \%$, while women's death rate was $1.7 \%$.

As of April 2020, it is not known whether past infection provides effective long-term immunity to those people who have recovered from COVID-19. Based on what it is know about other types of coronaviruses it is expected that recovered people would have some kind of immunity, yet available results are not completely conclusive.

\subsection{A topological model of COVID-19}

In this section we develop the main result of this essay: a topological model for the COVID-19 pandemic, associated to the sequence of Figures 2 to 11. Even though it will require from the reader to go back and forth, we will keep this sequence of images altogether to stress they constitute an conceptual unity that, from top to bottom, shows the evolution of the topology of the population $\mathrm{X}$ as the epidemic evolves. Each single figure represents a topological partition [20] of the set X of all citizens inhabiting in a given territory. It is worth to recall that the subsets of a partition are disjoint.

\subsubsection{The World before COVID-19.Based on the} information about COVID-19 collected above, we will assume that by 15 November 2019 COVID-19 did not exist at all. So, the whole population X was healthy, then susceptible $(\mathrm{S})$ in the nomenclature of basic SIR epidemiological models [24]. $\mathrm{T}=\{\Phi, X\}$, where $\Phi$ denotes the empty set, defines a topology for X. Then, the pair $(\mathrm{X}, \mathrm{T})$ is a topological space [20]. In this case, the whole population $X$ and the susceptible population $S$ coincide, and we will redundantly rewrite the topology as $\mathrm{T}=\{\Phi, \mathrm{X}, \mathrm{S}\}$ to remark that the susceptible population $\mathrm{S} \subseteq \mathrm{X}$ is a subset of the whole population. Figure 2 is a visual metaphor of the topological structure of the set $\mathrm{X}$ before the COVID-19 epidemic. The blue rectangle represents $\mathrm{X}$ and its partition $\mathrm{X}=\mathrm{S}$, consisting of one subset.
This topological model of COVID-19 says nothing about the dynamics of the population $\mathrm{X}$ before the outbreak of the epidemic, because the topological's and the dynamical's are different types of problems, living in different realms; this is so true, that several different, non-topologically equivalent, dynamical systems should be used to model the dynamics of $X$, depending on the properties $X$ should have. Thus, if we pose the problem within the usual short-term context of epidemiology, where the time-span of epidemics is supposed to be much shorter than the live spans of individuals, and the global populations should remain constant, a good model for the dynamics of $S$ would be $\dot{S}=0, S(0)=S_{0}$, and then the trajectories of the susceptible population would be $\mathrm{S}(\mathrm{t})=\mathrm{S}_{0}, \forall \mathrm{t} \geq 0$. On the contrary, if we would like to study the long-term evolution of population $X$, we would need to use a nonlinear model like $\dot{S}=\alpha \mathrm{S}-\beta \mathrm{S}^{2}, \mathrm{~S}(0)=\mathrm{S}_{0}$, to describe the dynamics of the system, which among other properties has two equilibrium points: a repellor located at $S=0$, and an attractor at $S=\alpha / \beta$. There is, however, a very important connection between the topological and the dynamical models, a kind of ceiling or upper bound the topology imposes to the dynamics: the order of the dynamics will at most be $N-1$, where $N$ is the number of opens of the partition of $X$. In this case $N=2$, then the order of the system is at most 1 , and the trajectories are curves $\varphi$ : $\mathbb{R} \times \mathbb{R} \rightarrow \mathbb{R},(\mathrm{t}, \mathrm{x}) \rightarrow \varphi(\mathrm{t}, \mathrm{x}) \in \mathbb{R}$, whose images are subsets of the one-dimensional phase-space $\mathbb{R}$.

2.2.2 The outbreak of COVID-19. It is not yet clear when, where, and how the SARS-CoV-2 started infecting humans; yet, humankind knew about this new strain of the CoV family, after the first symptomatic infected people appeared in the hospitals of Wuhan at the beginning of December 2019. So, for the sake of this essay, we will assume COVID-19 appeared and started infecting people sometime between 15 November and 1 December 2019, remaining undetected until the first symptomatic patients appeared. This situation is illustrated in Figure 3, where the blue and purple subsets represent the healthy susceptible and the asymptomatic infected people, respectively. Unfortunately, there is still no practical way to make a sharp cut distinction between healthy and asymptomatic people, and consequently all of them are taken as healthy people. Under this conditions of incertitude, the whole population $\mathrm{X}$ is treated as a single group covered by the topology $T=\{\Phi, \mathrm{X}, \mathrm{S}\}$.

2.2.3 First symptomatic patients appear. From the topological point of view, the documented infection of the very first patient represents a qualitative change in 
the topological structure of $\mathrm{X}$, because automatically $T=\{\Phi, X\}$ stops being a cover for $\mathrm{X}$, and the topology $\mathrm{T}$ must be refined to $\mathrm{T}_{1}=\left\{\Phi, \mathrm{X}, \mathrm{S}_{1}, \mathrm{I}_{1}\right\}$, where $\mathrm{S}_{1}$ and $\mathrm{I}_{1}$ represents the subset of susceptible and infected people, respectively, and $X=S_{1} I_{1}$. This is described in Figures 4 and 5, where the red blocks represent the subsets of infected people. A yellow block is also included in the visual metaphor to represent an intrinsically immune set of people that will never get infected by the coronavirus COVID-19. That such a group of people exists is a subject of present debate [25]. If we include the immune subset of people in the topology for $X$ we obtain $\mathrm{T}_{1}=\left\{\Phi, \mathrm{X}, \mathrm{Y}, \mathrm{S}_{1}, \mathrm{I}_{1}\right\}$, where $\mathrm{X}=\mathrm{YUS} \mathrm{UI}_{1}$. Consistently with previous discussion in sub subsection 2.2.1, the arrival of the coronavirus COVID-19 generated an internal explosion in $X$, that refined its original topology $\mathrm{T}=\{\Phi, \mathrm{X}, \mathrm{S}\}$ into $\mathrm{T}_{1}=\left\{\Phi, \mathrm{X}, \mathrm{Y}, \mathrm{S}_{1}, \mathrm{I}_{1}\right\}$, and could in turn transform the original one-dimensional trajectories $\varphi: \mathbb{R} \times \mathbb{R} \rightarrow \mathbb{R}$ into bi or tridimensional trajectories $\varphi$ : $\mathbb{R} \times \mathbb{R}^{n} \rightarrow \mathbb{R}^{n}$, with $\mathrm{n}=\{2,3\}$. The properties of these extended trajectories would essentially be determined by the type of interaction occurring between the subpopulations $\mathrm{Y}, \mathrm{S}_{1}$, and $\mathrm{I}_{1}$.

To illustrate the point, let us suppose that the interaction between the susceptible population $\mathrm{S}_{1}$ and coronavirus COVID-19 that generates the infected people $\mathrm{I}_{1}$ is given by the basic SIR model [24]

$$
\left(\begin{array}{c}
\dot{s} \\
I \\
\dot{R}
\end{array}\right)=\left(\begin{array}{c}
-\beta S I \\
\beta S I-\alpha I \\
\alpha I
\end{array}\right)
$$

If one think of the SIR model like a control system, it is obvious that it is a second order system, despite containing three differential equations. In fact, the third equation is a kind of integrable output equation whose integration allows expressing the recovered population in terms of the infected population: $\mathrm{R}(\mathrm{t})=\mathrm{R}(0)+\alpha \int_{0}^{\mathrm{t}} I(s)$ $d s$.

The independence of the first two equations with respect to the recovering output function $R(t)$ indicates that, according to this particular kind of SIR model, once people overcome the infection by COVID-19 they will not get reinfected by the same coronavirus; so, the lack of feedback loops of the output signal $\mathrm{R}(\mathrm{t})$ into the differential equations modeling the dynamics of the susceptible and the infected populations is, by itself, a model of permanent adquired immunity to COVID-19.

2.2.4 First COVID-19 deaths. In Figure 6 two important landmarks are described: the infected population reaches its peak, and the first deaths occur. Topologically, this means the topology $\mathrm{T}_{1}=\left\{\Phi, X, Y, S_{1}, I_{1}\right\}$ exploded into $\mathrm{T}_{2}=\left\{\Phi, X, Y, S_{2}, I_{2}, M_{1}\right\}$, where $M_{1}$ represents the gray open subset in Figure 6. The monotonic increasing population $\mathrm{M}$ could be modeled by a second algebraic output equation $M(t)=I(t)-R(t)$, which does not change the order of the system.

2.2.5 Infected people recover. Figures 7 to 9 model the explosions of the topology $T_{2}=\left\{\Phi, X, Y, S_{2}, I_{2}, M_{1}\right\}$ for $\mathrm{X}$ into the finer topologies $\mathrm{T}_{\mathrm{i}}=\left\{\Phi, \mathrm{X}, \mathrm{Y}, \mathrm{S}_{\mathrm{i}}, \mathrm{I}_{\mathrm{i}}, \mathrm{M}_{\mathrm{i}-1}, \mathrm{R}_{\mathrm{r}}\right.$ $\left.{ }_{2}, \mathrm{R}_{\mathrm{i}-2}^{1}\right\}, 3 \leq \mathrm{i} \leq 5$, where $\mathrm{R}_{\mathrm{i}-2}^{\mathrm{d}}, \mathrm{R}_{\mathrm{i}-2}^{1}$ represent the dark and light green subsets of recovered people with and without immunity, respectively. The topologies $T_{i}, 3 \leq i \leq 5$, are all three structurally equivalent, yet they are included to suggest that epidemics could evolve manifoldly, both topologically and dynamically. Topologies and dynamics complement each other: the topological approach is better fitted to global thinking, planning and deciding, while the dynamical approach excels at detailed analysis, quantitative predictions, and control.

The SIR model (1) represents recovering with permanent afterwards immunity. Were the immunity transitory, the dark green open set in the partition in Figure 10 would evolve into a blue open set, instead of a yellow one, in the topology in Figure 11. In this case, the model (1) should be replace by another one permitting to feed the output signal $\mathrm{R}$ back into the differential equations for $\mathrm{S}$ and $\mathrm{I}$, to modify their dynamics accordingly.

2.2.6 The first round of COVID-19 ends. Topologically speaking, Figures 2 to 11 suggest that COVID-19 can be thought of as a finite sequence of refinements of a topological partition for the set X, starting with the indiscrete topology, and ending with the partition $\mathrm{T}_{5}$. The first round of COVID-19 ends when the sequence of topological explosions in the topology for X stops, and a reverse process of implosions starts, as in Figures 10 and 11 , where first $\mathrm{T}_{5}=\left\{\Phi, X, Y, S_{5}, I_{5}, M_{4}, \mathrm{R}_{3}^{d}, \mathrm{R}_{3}^{l}\right\}$ implodes into $\mathrm{T}_{6}=\left\{\Phi, X, Y, S_{6}, M_{5}, \mathrm{R}_{4}^{d}, \mathrm{R}_{4}^{l}\right\}$, Figure 10 , then $\mathrm{T}_{6}$ implodes first into $\mathrm{T}_{7}=\left\{\Phi, X, Y, S_{7}, M_{6}, \mathrm{R}_{5}^{d}\right\}$, and then $\mathrm{T}_{7}$ implodes into $\mathrm{T}_{8}=\left\{\Phi, X, Y_{1}, S_{8}, M_{7}\right\}$, Figure 11, where $Y_{I}=$ $Y \otimes R_{5}^{d}$.

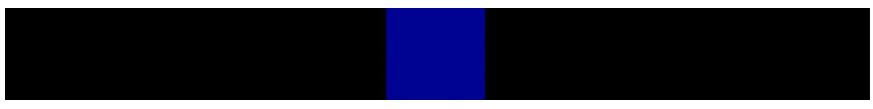

Figure 2. The world before COVID-19 pandemic.

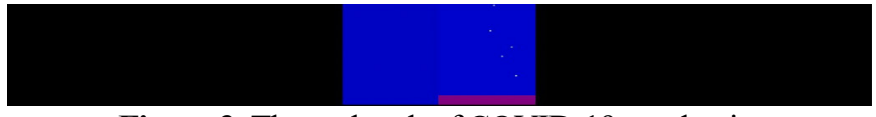

Figure 3. The outbreak of COVID-19 pandemic. 


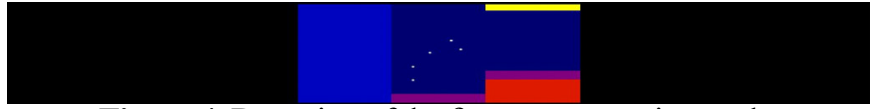

Figure 4. Detection of the first symptomatic people.

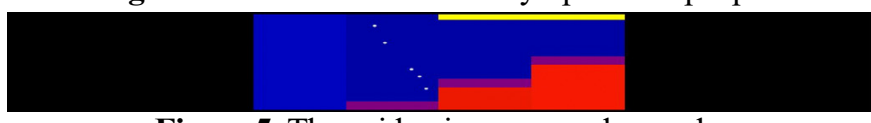

Figure 5. The epidemic grows and spreads.

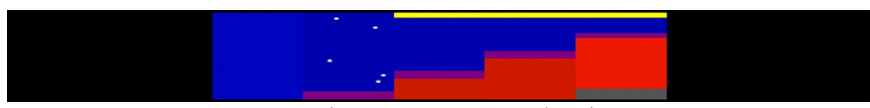

Figure 6. First COVID-19 deaths occur.

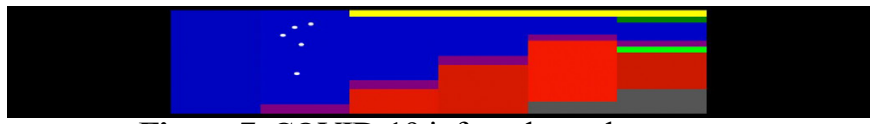

Figure 7. COVID-19 infected people recover.

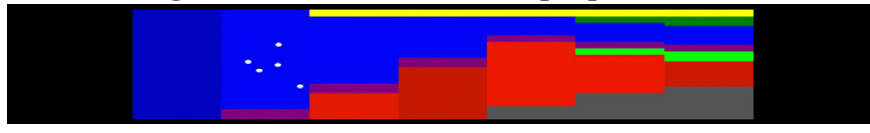

Figure 8. COVID-19 infection keeps decreasing.

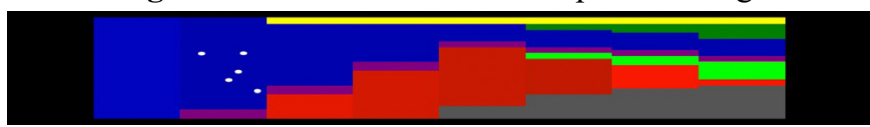

Figure 9. COVID-19 recovering keeps increasing.

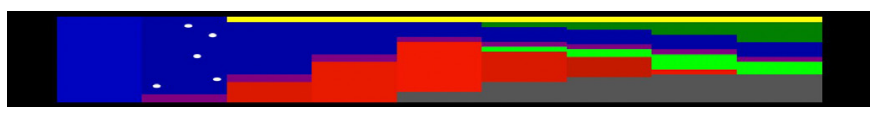

Figure 10. COVID-19 infection ends.

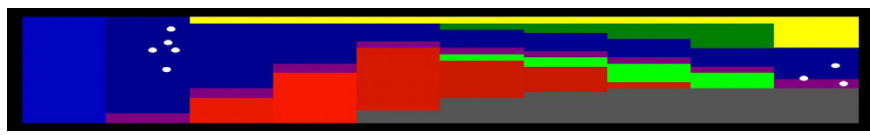

Figure 11. Ready for a second round of COVID-19?

\section{Conclusions}

In this essay we developed a topological model that provides a qualitative tool to describe the main features of COVID-19 pandemic. This topological model clearly separates topological from dynamical events in the outbreak of an epidemic, showing that topological transformations of the partition of the phase-space $\mathrm{X}$ always preceded the external dynamic manifestations of the epidemic. That sharp-cut topological events precede all drastic changes in the qualitative behavior of dynamical systems seem to be universal, which could help to foresee and classify normal and anomalous scenarios, dynamics, and operation modes in dynamical processes of different nature. Even though topology is seldom use as a tool for systems modeling, that it could demand an unusual level of abstraction or a different kind of mental training from the modeler, from the point of view of the users it only requires a good level of qualitative understanding of the processes to be modeled. The reported topological model could be easily extended to study more complex systems and scenarios, and can be naturally transformed into stop-motions for didactic purposes. From top to bottom Figures 2 to 11 describe a sequence of topological transformations of the phase-space $\mathrm{X}$, while from left to right show parametrized sequences of events that alter the dynamics of a dynamical system.

\section{References}

[1] S. P. Huntington. The Clash of Civilizations and the Remaking of the World Order. New York: SIMON \& SCHUSTER, 2003.

[2] F. Brauer, "Mathematical epidemiology: Past, present, and future," Infectious Disease Modelling, 2, pp. 113-127, 2017. https://doi:10.1016/j. idm.2017.02.001.

[3] M. Hirsch and S. Smale. Differential Equations, Dynamical Systems, and Linear Algebra, New York, Academic Press, 1974.

[4] V. I. Arnol'd, Ordinary Differential Equations, Berlin, Springer-Verlag, 1992.

[5] K. S. Sibirsky, Introduction to topological dynamics, Leyden, Noordhoff International Publishing, 1975.

[6] H. Rago, Brevísima biografía. In H. Rago (Compiler), L. Herrera, C. Domingo, D. Morales, and A. Capelletti, Newton, pp. 13-23, Mérida, Universidad de Los Andes, 2005.

[7] H. Rago and L. Herrera. Newton y el universo físico. In H. Rago (Compiler), L. Herrera, C. Domingo, D. Morales, and A. Capelletti, Newton, pp. 25-96, Mérida, Universidad de Los Andes, 2005.

[8] M. Farkas and M. Pidal, Estabilidad estructural y bifurcaciones, Caracas, Universidad Central de Venezuela, 1981.

[9] D. K. Arrowsmith and C. M. Place, An introduction to dynamical systems, Cambridge, Cambridge University Press, 1990.

[10] J. Hale. Ordinary Differential Equations, New York, Wiley-Interscience, 1969.

[11] E. Coddington and N. Levison, Theory of Ordinary Differential Equations, New Delhi, Tata McGraw-Hill, 1977.

[12] P. Hartman. Ordinary Differential Equations, Second Edition, Boston, Birkhäuser, 1982.

[13] V. V. Nemytski and V. V. Stepanov. Qualitative Theory of Differential Equations, Fourth Printing, Princeton, Princeton University Press, 1972.

[14] L. Elsglotz, Ecuaciones Diferenciales y Cálculo Variacional, Moscú, MIR, 1969.

[15] F. Verhulst. Nonlinear Differential Equations and Dynamical Systems, Berlin, Springer-Verlag, 1990.

[16] L. Perko. Differential Equations and Dynamical 
Systems, Second Edition, New York, Springer-Verlag, 1996.

[17] H. Amann. Ordinary Differential Equations - An Introduction to Nonlinear Analysis, Berlin, Walter de Gruyter, 1990.

[18] J. Dugundji. Topology, Boston, Allyn and Bacon, Inc., 1966.

[19] J. Munkres. Topology: A First Course, Englewood Cliffs, Prentice-Hall, 1975.

[20] J. Rodríguez-Millán. "A topological approach to designing and constructing dynamical visual metaphors of multicultural and intercultural systems II-A", Revista Ciencia e Ingeniería, Vol. 41, no. 1, pp. 111-122, diciembre-marzo, 2020.

[21] J. Rodríguez-Millán. "A topological approach to designing and constructing dynamical visual metaphors of multicultural and intercultural systems I", Revista Ciencia e Ingeniería, Vol. 40, no. 3, pp. 253-260, agosto-noviembre, 2019.

[22] D. Cyranoski. Profile of a Killer Virus, Nature, Vol. 581, pp. 23-26, 7 May, 2020.

[23] John Hopkins University of Medicine, COVID-19 Dashboard by the Center for Systems Science and Engineering, https://coronavirus.jhu. edu/map.html.

[24] F. Brauer. Compartmental Models in Epidemiology, In F. Brauer, P. van den Driessche, and J. Wu (Editors), Mathematical Epidemiology, pp. 1978, Berlin, Springer-Verlag, 2008.

[25] A. Sette and S. Crotty. Pre-existing immunity to SARS-CoV-2: the knowns and unknowns, Nature Review/Immuno- logy, https://doi.org/10.1038/ s41577-020-0389-z, (2020) 